Article

\title{
A Hybrid End-to-End Control Strategy Combining Dueling Deep Q-network and PID for Transient Boost Control of a Diesel Engine with Variable Geometry Turbocharger and Cooled EGR
}

\author{
Bo Hu* (D), Jiaxi Li, Shuang Li and Jie Yang \\ Key Laboratory of Advanced Manufacturing Technology for Automobile Parts, Ministry of Education, \\ Chongqing University of Technology, Chongqing 400054, China; 11607990404@2016.cqut.edu.cn (J.L.); \\ 1s123456@2016.cqut.edu.cn (S.L.); j.young@2017.cqut.edu.cn (J.Y.) \\ * Correspondence: b.hu@cqut.edu.cn
}

Received: 12 September 2019; Accepted: 29 September 2019; Published: 30 September 2019

\begin{abstract}
Deep reinforcement learning (DRL), which excels at solving a wide variety of Atari and board games, is an area of machine learning that combines the deep learning approach and reinforcement learning (RL). However, to the authors' best knowledge, there seem to be few studies that apply the latest DRL algorithms on real-world powertrain control problems. If there are any, the requirement of classical model-free DRL algorithms typically for a large number of random exploration in order to realize good control performance makes it almost impossible to implement directly on a real plant. Unlike most of the other DRL studies, whose control strategies can only be trained in a simulation environment-especially when a control strategy has to be learned from scratch - in this study, a hybrid end-to-end control strategy combining one of the latest DRL approaches-i.e., a dueling deep Q-network and traditional Proportion Integration Differentiation (PID) controller-is built, assuming no fidelity simulation model exists. Taking the boost control of a diesel engine with a variable geometry turbocharger (VGT) and cooled (exhaust gas recirculation) EGR as an example, under the common driving cycle, the integral absolute error (IAE) values with the proposed algorithm are improved by $20.66 \%$ and $9.7 \%$ respectively for the control performance and generality index, compared with a fine-tuned PID benchmark. In addition, the proposed method can also improve system adaptiveness by adding another redundant control module. This makes it attractive to real plant control problems whose simulation models do not exist, and whose environment may change over time.
\end{abstract}

Keywords: hybrid control strategy; dueling deep Q-network; PID; transient boost control; VGT

\section{Introduction}

Turbocharging and boosting are key technologies in the continued drive for improved internal combustion engine efficiency with reduced emissions [1]. For more than a decade, engine boosting has seen widespread adoption by passenger and heavy goods vehicle powertrains in order to increase the specific power and enable the downsizing megatrend [2]. However, many challenges still remain, as the regulation requirements become stricter, and the demand for low-carbon powertrains increases [3]. Currently, the growing expectations of vehicle performance, including an excellent transient response with high boost levels, have converged within the demand for increased downsizing and higher levels of EGR. For boosting machinery, the rated power and torque for downsized units are conventionally regained via fixed-geometry turbocharging [4]. However, the transient behavior of such systems is limited by the usual requirement of a large size turbocharger, especially if a high-end torque is 
pursued [5,6]. VGT technology (see Figure 1), which is designed to vary the effective aspect ratio of the turbocharger under different engine operating conditions [7], can significantly improve an engine's transient response and fuel economy compared with a fixed-geometry turbocharger [8].

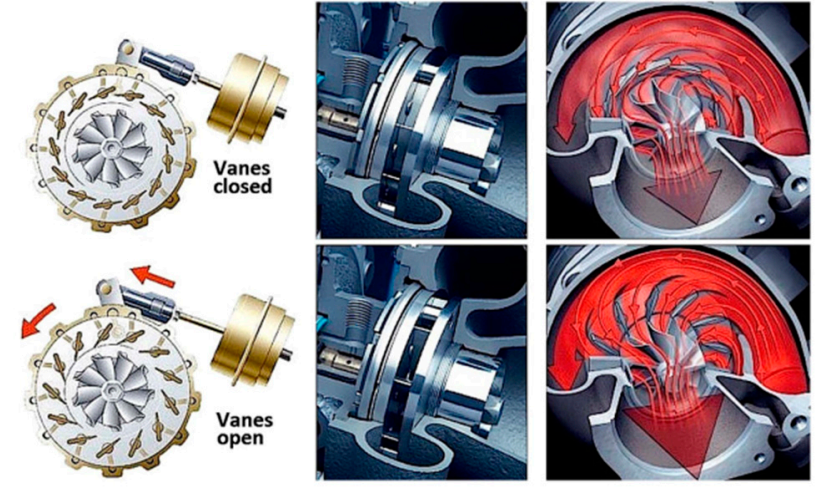

Figure 1. Variable geometry turbocharger (VGT) operating principle [7].

Due to the nonlinear characteristics of the VGT system and the fact that EGR and VGT systems are strongly interactive, the boost control of the VGT is recognized as a major challenge for diesel engines [9]. Currently, the fixed-parameter gain-schedule PID control is used in the automotive industry for VGT boost control owing to its simplicity, robustness, and effectiveness [10], but the control performance is sensitive to the state of the control loop, and is difficult to be satisfactory when the loop alters $[11,12]$. One of the feasible approaches to solve this problem is by proposing adaptive hybrid PID controllers. For example, in [13], the fuzzy technique was combined with a PI controller, and a better control performance was demonstrated. In addition, Sant and Rajagopal proposed a hybrid control system that includes a steady-state PI controller and a transient fuzzy controller [14]. However, both these approaches adopt offline-tuning rules, which are sensitive to system uncertainty. Another direction to improve the behavior of a PID controller is to replace it with a brand-new control structure. For example, an online self-learning deep deterministic policy gradient (DDPG) algorithm was employed for the boost control of a VGT-equipped engine. Although the proposed strategy can develop good transient control behavior by direct interaction with its environment, it takes much time for the algorithm to learn from no experience, and it is hardly possible to train the algorithm directly on a real plant due to its random exploration when a control strategy has to be learned from scratch [15].

Reinforcement learning (RL), being as one of three machine learning paradigms, along with supervised learning and unsupervised learning, is the field of machine learning focusing on how control actions are selected in the environment in an optimal manner by trial and error [16,17]. The theory of reinforcement learning, inspired by the psychology of behaviorism, focuses on online learning and tries to maintain a balance between exploration and exploitation [18]. Different from supervised learning and unsupervised learning, reinforcement learning does not require any pre-given data, but obtains learning information and updates model parameters by receiving rewards (feedback) from the environment for actions [19-21]. Deep reinforcement learning (DRL), which excels at solving a wide variety of Atari and board games, is an area of machine learning that combines the deep learning approach and reinforcement learning (RL) [22,23]. The deep Q-network (DQN) strategy adopted on AlphaGo [24] and AlphaGo Zero [25] formed the first computer program that defeated human experts at the game of Go. It effectively solves the problem of instability and divergence caused by the use of neural network nonlinear value approximators through experience replay and fixed Q-Target, which greatly improves the applicability of reinforcement learning. Recently, Wang et al. [26] updated DQN by proposing an architecture that consists of dueling networks. Their dueling networks replace the state-action value by two separate estimators: one for state-value function, and the other for state-dependent advantage function. This is particularly beneficial if a control action does not have any influence on the environment in any situation. By doing so, their RL agent proved superior 
to the latest technologies in the Atari 2600 domain. However, the aforementioned model-free DRL algorithms typically require a very large number of random exploration before achieving a good control performance; thus, it is hardly possible to apply the algorithm directly on a real plant, and have to rely heavily on a simulation environment, especially when a control strategy has to be learned from scratch. In contrast, the traditional PID method can control a process from its beginning to its end, and deliver a complete function solution without needing to procure anything from a third party (i.e., the concept of end-to-end); therefore, it can be easily implemented on real controllers without relying on simulation models. Based on the discussion above and that there exists no simulation model at many times for the training of a pure DRL strategy, it is interesting to combine a traditional PID controller and an intelligent DRL strategy together in order to realize an end-to-end control using the latest DRL achievement in a real environment.

In the following paper, a hybrid end-to-end control strategy combining an intelligent dueling deep Q-network and traditional PID for the transient boost control of a diesel engine with a variable geometry turbocharger and cooled EGR will be proposed. The remaining of this article is structured as follows: In Section 2, the hybrid control framework is proposed to realize an optimal boost control of a VGT-equipped engine. In Section 3, a comparison between the proposed end-to-end hybrid algorithm, a classical model-free DRL algorithm, and a fine-tuned PID controller are conducted and discussed. Section 4 concludes the article.

\section{Hybrid Control Framework}

In this section, firstly, the mean value model of the research engine is introduced. After that, the implementation mechanism of the dueling DQN and the corresponding testing platform are described. Finally, the proposed adaptive hybrid control strategy combining intelligent DQN and traditional PID is elaborated.

\subsection{Engine Model Analysis}

In this article, the boost control strategy is implemented on a six-cylinder, three-liter VGT-equipped diesel engine, which can be seen in Figure 2. A detailed engine model has been converted to a mean value model in order to reduce the run time without sacrificing transient accuracy [27] (see Ref. [15] for detailed explanation). Note that the mean value GT-Suite model (see Figure 3) serves as a "real" engine environment in this study, so as to show the results between the end-to-end and non-end-to-end model-free approaches, but the proposed method can be transferred to a real plant easily. In order to provide a comparison, the model is initially controlled by a fine-tuned PID controller.

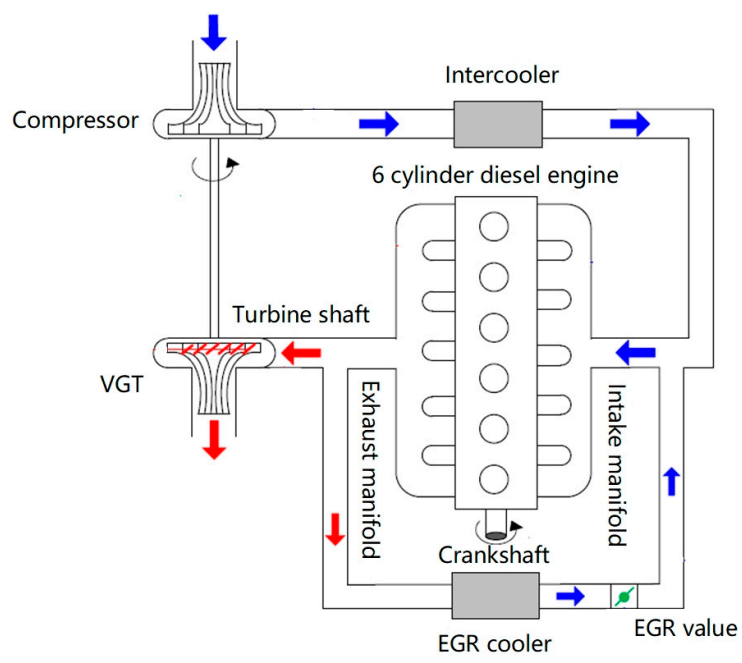

Figure 2. VGT-equipped diesel engine with exhaust gas recirculation (EGR) system. 


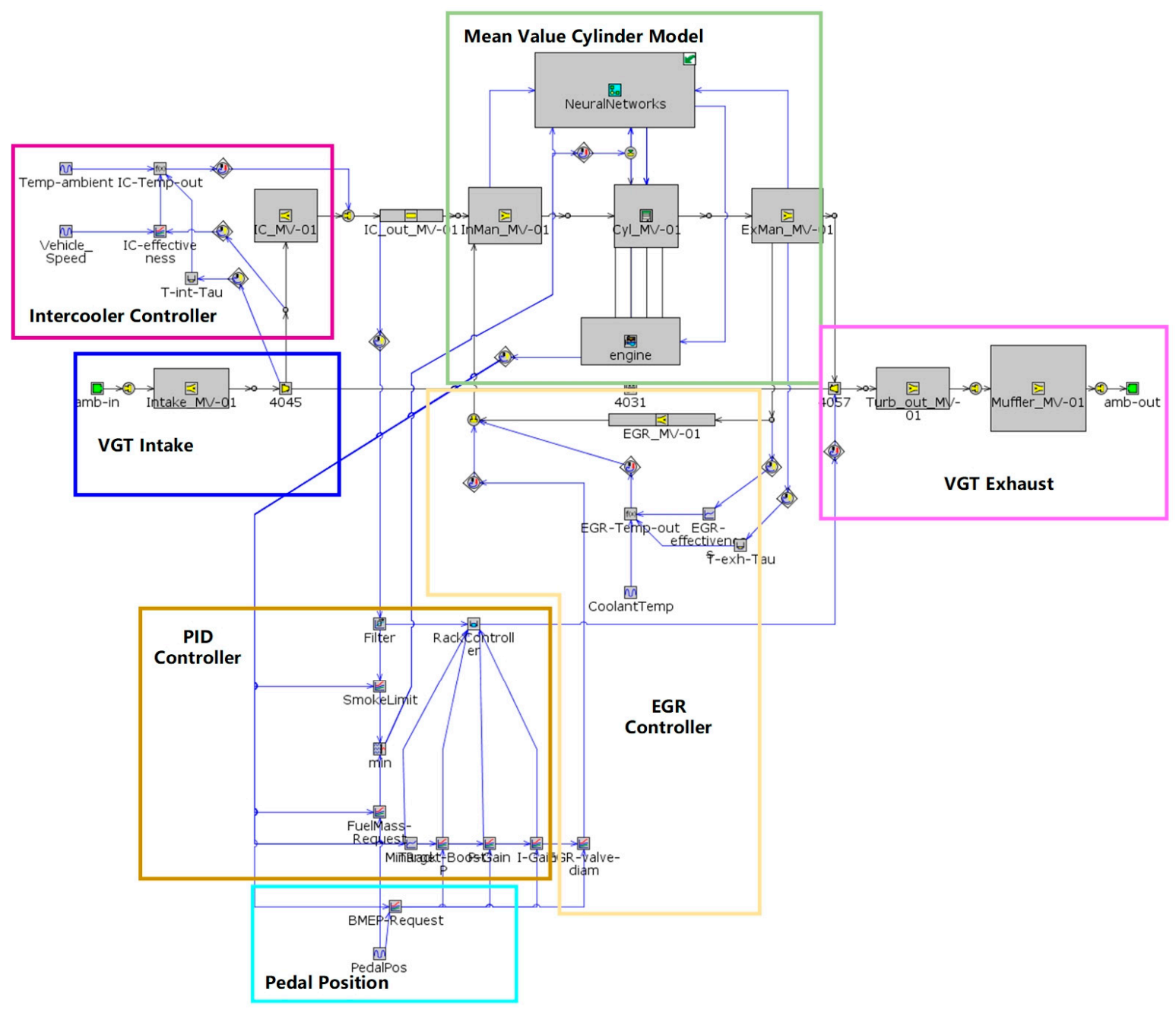

Figure 3. Layout of the GT-Suite mean value model.

\subsection{Dueling Deep Q-Network Architecture}

Reinforcement learning is a computational approach to learning whereby an agent tries to maximize the total amount of reward it receives when interacting with environment (see Figure 4) $[28,29]$. For every state $s$, the agents always try to maximize the expected discounted return by choosing an action $a$. The discounted return is defined as $R_{t}=\sum_{\tau=t}^{\infty} \gamma^{\tau-t} r_{\tau}$, where $\gamma \epsilon[0,1]$ is a discount factor and $r$ stands for the reward that is observed by the agent after an action is exerted on the environment.

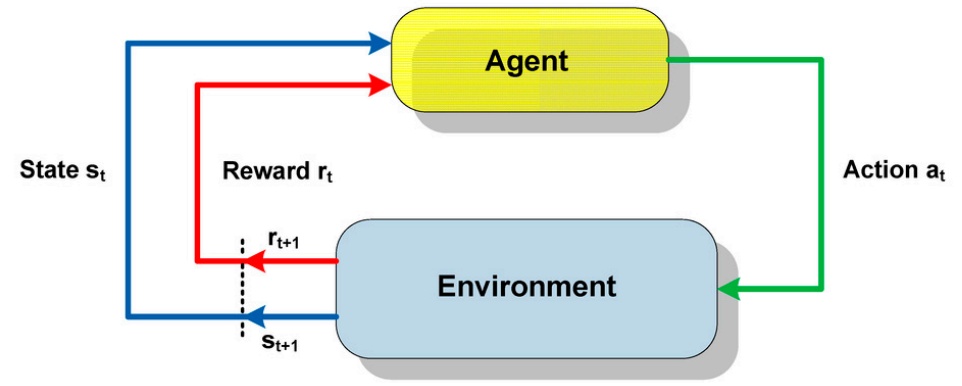

Figure 4. Basic idea and elements involved in a reinforcement learning formalization.

For an agent behaving according to a policy $\pi$, the state-action function and the state function are defined as follows:

$$
Q^{\pi}(s, a)=\mathbb{E}\left[R_{t} \mid s_{t}=s, a_{t}=a, \pi\right]
$$


and:

$$
V^{\pi}(s)=\mathbb{E}_{a \sim \pi(s)}\left[Q^{\pi}(s, a)\right]
$$

The value function described above is a high-dimensional object. For approximation, a deep Q-network $Q(s, a ; \theta)$ with parameter $\theta$ can be used, and the following loss function sequence is optimized at iteration $i$ :

$$
L_{i}\left(\theta_{i}\right)=\mathbb{E}_{s, a, r, s^{\prime}}\left[\left(y_{i}^{D Q N}-Q\left(s, a ; \theta_{i}\right)\right)^{2}\right]
$$

In order to solve the problem of instability and divergence caused by the use of nonlinear value approximators, the classical DQN algorithm adopted the strategy of experience replay and fixed Q-target, which are considered as two key factors in its great success on Alpha Go. The following shows the algorithm pseudo-code of the proposed DQN algorithm for the boost control problem in this study.

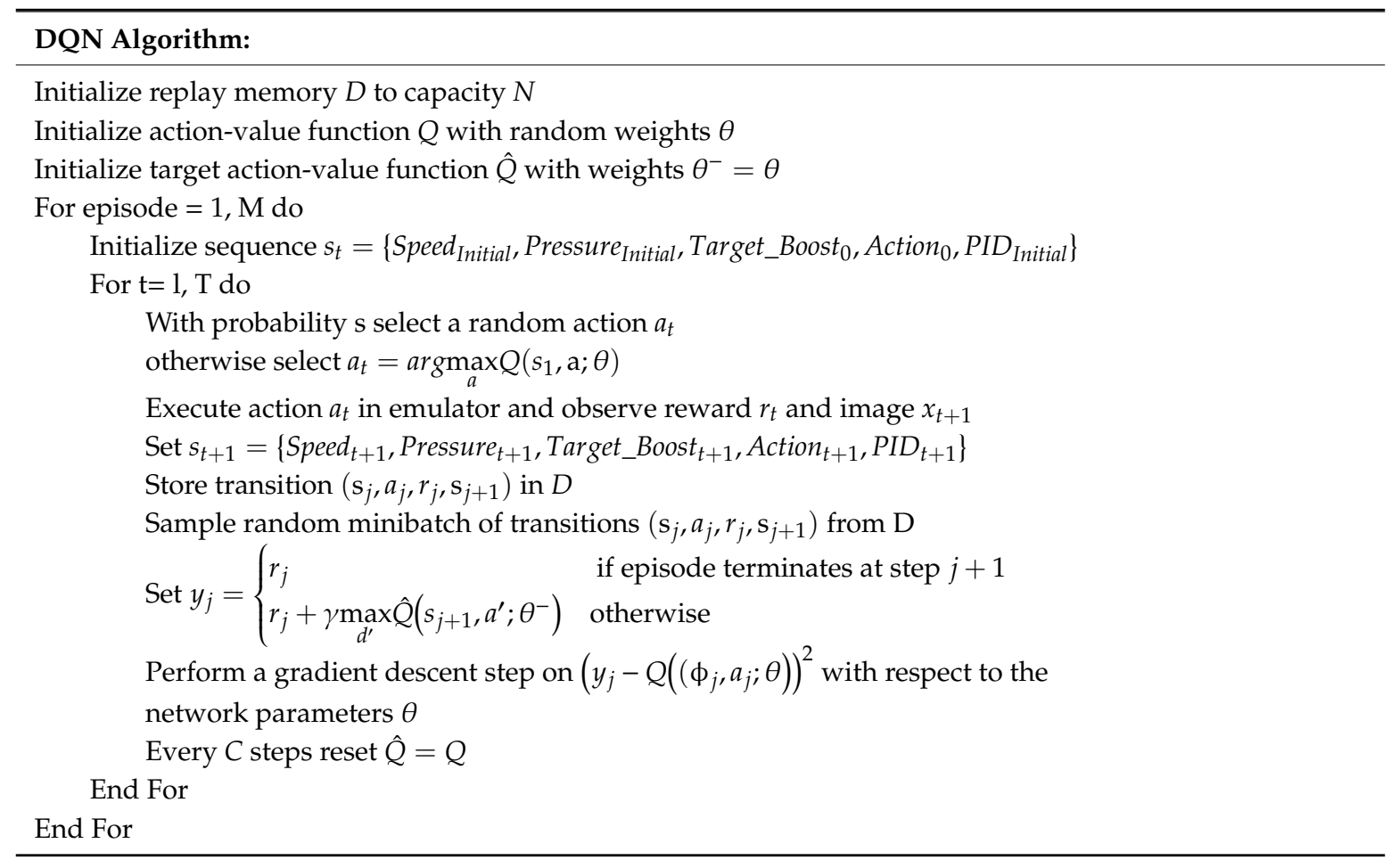

The updated dueling DQN algorithm implementation and the corresponding testing platform are illustrated in Figure 5. Unlike the classical DQN, which only produces a single output $Q$ function, the dueling DQN use two streams of fully connected layers to provide the estimate of the state and the advantage functions before combining them to generate output $Q$ functions. By separating the state value function from the $Q$ function, the updated dueling $D Q N$ is capable of solving control problems whose estimate of the state values is of great importance, while for many circumstances, the choice of actions does not have any influence on the environment. The vane position controlled by a membrane vacuum actuator is selected for the control action, and the four quantities of engine speed, actual boost pressure, target boost pressure, and current vane position are used to group the four-dimensional state space. For the choice of immediate reward $\left(r_{t}\right)$, the function of the error between the target boost and the current boost $(e(t))$ plus the action change rate $\left(I_{t}\right)$ is defined, and the specific formula is as follows:

$$
r_{t}=e^{-\frac{\left[0.95 *|e(t)|+0.05 *\left|I_{t}\right|\right]^{2}}{2}}-1
$$




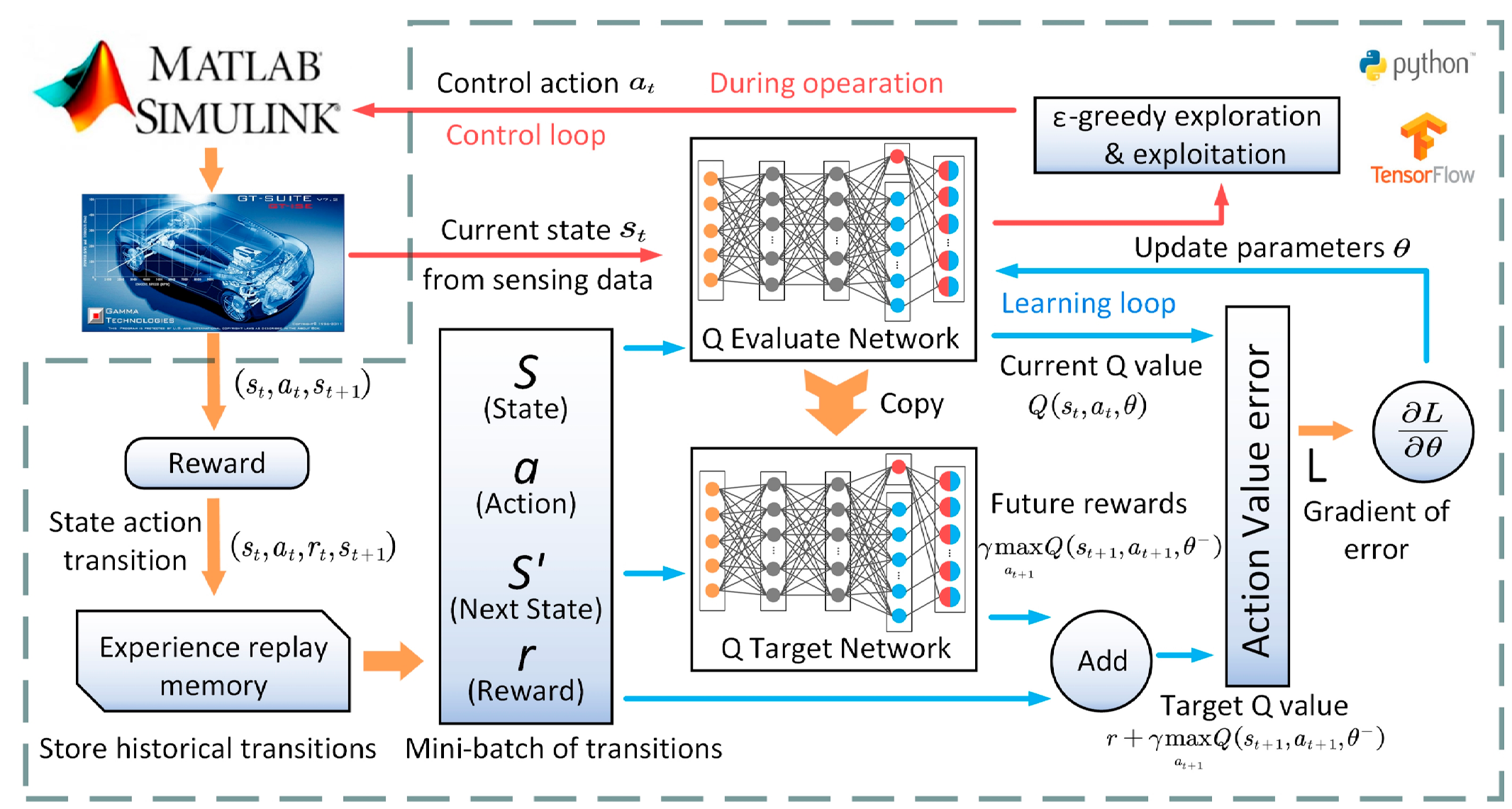

Figure 5. Dueling deep Q-network (DQN) implementation mechanism and the corresponding testing platform. 
The dueling Q-network illustration and the parameters of the proposed dueling DQN can be seen from Figure 6 and Table 1. In this study, the input layer of the dueling Q-network has five states, and there are two hidden layers each having 80 neurons, respectively. Before acquiring the $Q$ function at the output layer, two streams that independently estimate the state functions and the advantages functions are constructed. It should be noted here that the hyper parameters are only using standard values without fine tuning. Although in theory the control performance could be further improved, this is out of the scope of this research, as the objective of this paper is only to introduce another intelligent control frame to track the boost pressure for a VGT-equipped diesel engine. The training dataset is composed of 50 data episodes, and each episode represents the data for the first 1098s of the FTP-72 trips.

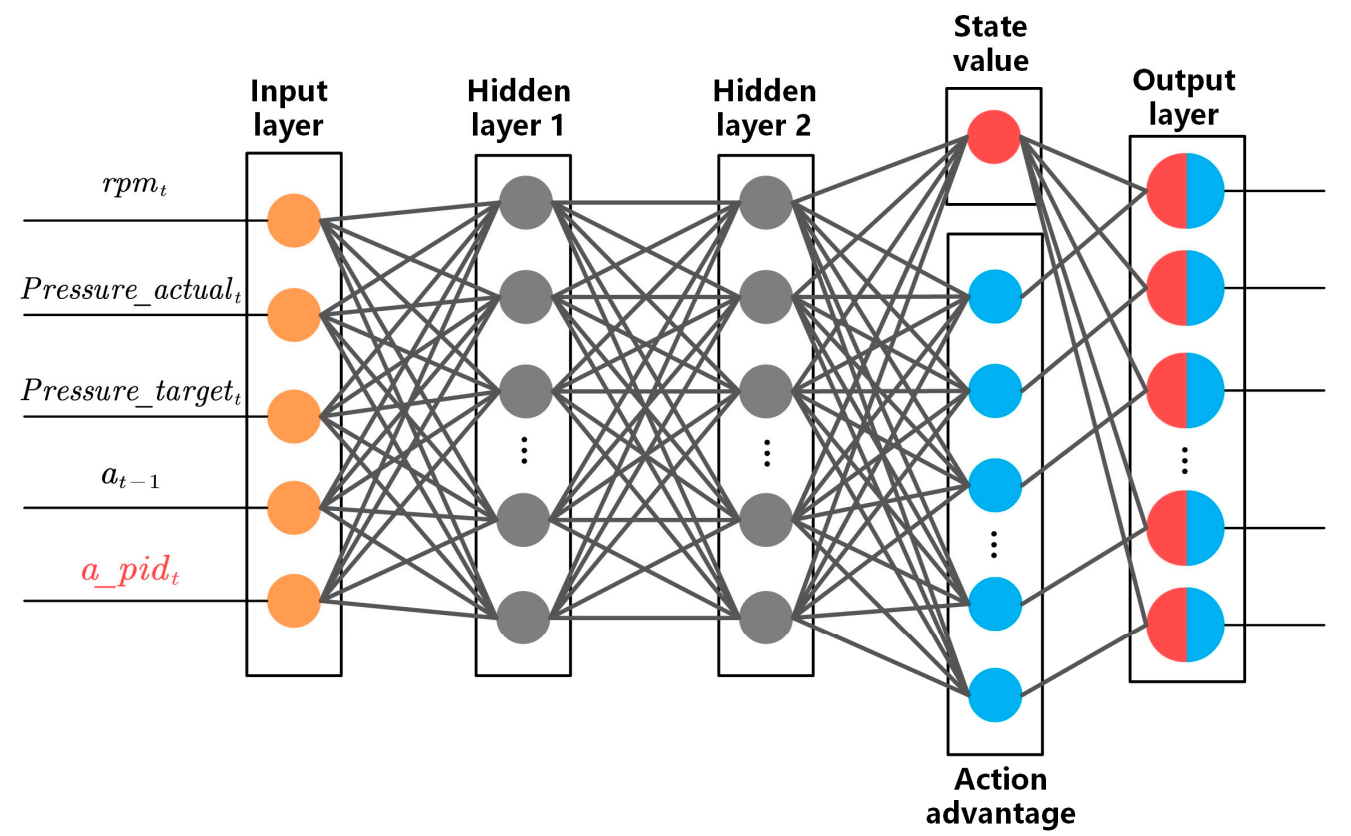

Figure 6. Illustration of the dueling Q-network.

Table 1. Dueling DQN parameters.

\begin{tabular}{cc}
\hline Parameters & Value \\
\hline Learning rate & 0.001 \\
Reward decay & 0.9 \\
Replay memory size & 10000 \\
Mini-batch size & 128 \\
$\mathcal{E}$-greedy & 0.95 \\
$\mathcal{\varepsilon}$-greedy increment & 0.000005 \\
\hline
\end{tabular}

\subsection{Adaptive Hybrid Control System Combining Intelligent Dueling DQN and Traditional PID}

Most of the control processes in the industry adopt the PID controller currently due to its simple structure and robust performance under complex conditions. However, the fixed-parameter structure makes it degraded when the control loop alters. Meanwhile, the DRL algorithm can self-learn a good control behavior by direct interaction with the environment. Therefore, it is interesting to establish a hybrid algorithm that combines the intelligent DRL (for example, the aforementioned dueling DQN) algorithm and a traditional PID controller, in order to take advantage of DRL's self-learning capability to tune a PID performance online. Unlike the practice proposed by some literatures [16,30], which uses the reinforcement learning approach to adjust the gains of PID controllers, in this paper, a simpler but more powerful method will be introduced by adding a dueling DQN algorithm directly after a fine-tuned PID controller, as can be seen in Figure 7. There are two special modifications that need to 
be considered here. First, the action range of the dueling DQN is limited, and for paper simplicity, this will be elaborated later. Second, another state-i.e., the PID action output—will be imported to the aforementioned dueling DQN algorithm. This is a practice to allow the dueling DQN strategy to learn the system behavior controlled by an existing PID controller.

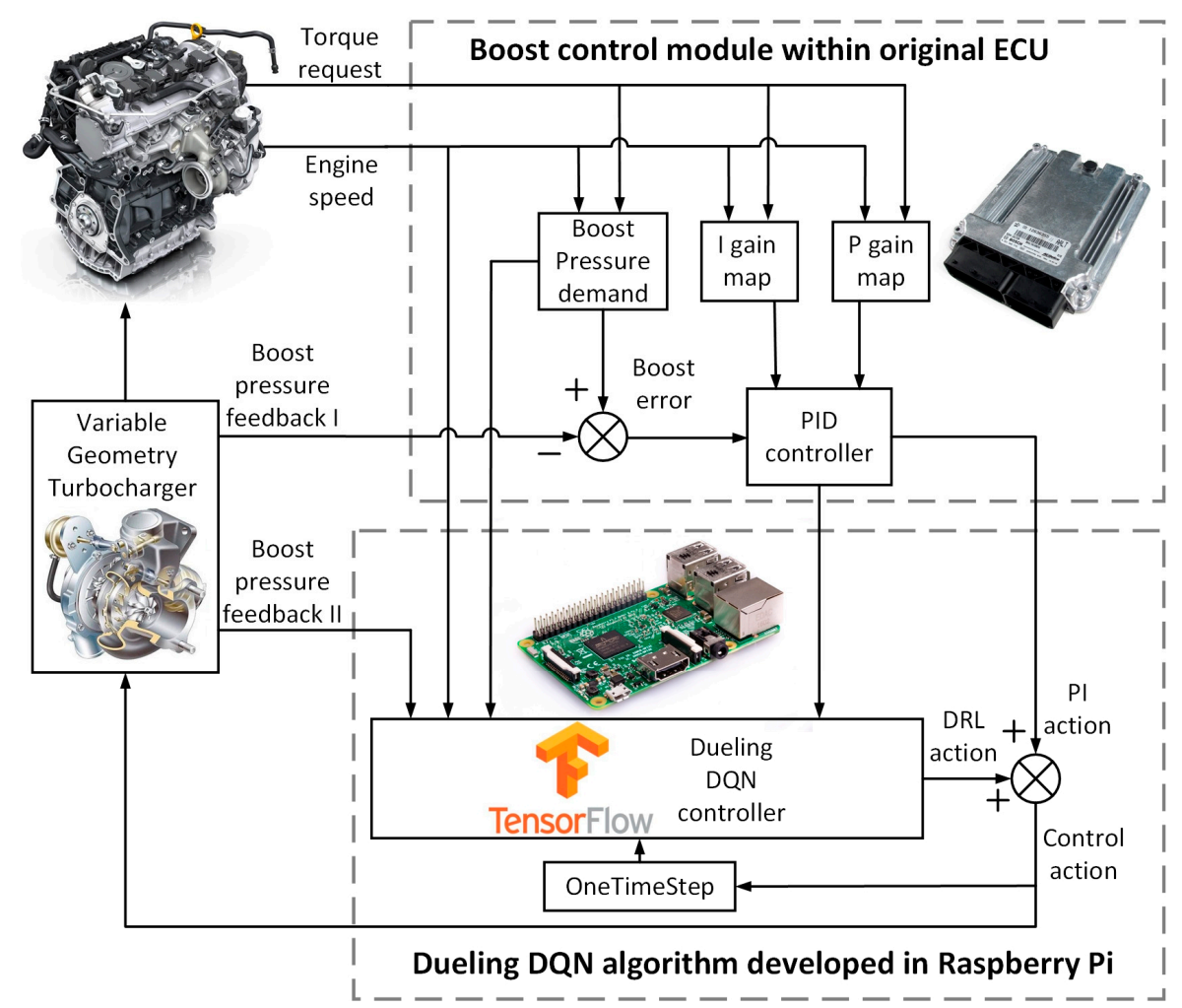

Figure 7. Adaptive hybrid control system schematic combining a dueling deep Q-network and traditional Proportion Integration Differentiation (PID) for a variable geometry turbocharger system.

This approach will intuitively enable a reasonable learning curve, as the proposed dueling DQN only needs to adjust an already fine-tuned PID output. However, the most innovative aspect of this practice is allowing the training process of the DRL algorithm to be performed directly for a real plant. It is known that 'conventional' DRL algorithms require a large number of random explorations especially at the beginning of the training and this, for most real plants, is not allowed, as poor action behaviors could cause damage to or even destroy the plants. Thus, for most RL study, a large part of the training (especially for the beginning) can only be carried out in a simulation environment. Depending on the fidelity of the simulation model, the control strategy obtained from the simulation may need to continue learning with the real environment. Although this method combines the simulation training and the experimental continuing training together in order to fully utilize the computational resources offline and refine the algorithm in the experimental environment online, a simulation model with relatively high fidelity is required. Thus, this approach is not 'model-free' in the strict sense. By combining both an intelligent dueling DQN and a traditional PID, this hybrid control approach, innovatively allowing the training process of the DRL algorithm that previously could only be carried out in the simulation environment, can now be performed directly on a real plant. This is realized by giving the control strategy some 'guided experience' before learning through making use of an existing fine-tuned PID controller and then allowing DRL control actions to autonomously learn the interaction with the real environment in order to further improve the control performance, based on a relatively good benchmark. With the help of the PID controller offering a good baseline, the combined control action will oscillate decently, even at the beginning of training, without violating real plant safety limit and not affecting the exploration performance of the algorithm. This approach, to the best 
of the authors' knowledge, will be the first attempt that is able to apply the DRL directly on a real plant, which in a sense achieves the real 'model-free' control.

\section{Results and Discussion}

The presented hybrid end-to-end control strategy is validated in this section. In order to mimic real-world driving behavior, the target boost pressure of the engine under a US FTP-72 (Federal Test Procedure 72) driving cycle was selected as the control objective, and the first $80 \%$ of this dataset is used to train the learning algorithm with the remaining for testing analysis. A comparative analysis of the tracking performance between a benchmark PID controller, a classical model-free DRL algorithm, and the dueling DQN + PID algorithm is performed to verify the advantage of the proposed method. First, the control performance with a fine-tuned gain-schedule PID controller is shown. Second, the learning curve of a classic model-free DRL algorithm and the proposed algorithm is discussed, and the boost tracking performance at the very first training episode for both algorithms is compared. Finally, the control performance of the proposed algorithm is demonstrated to achieve a high level of control performance, generality, and adaptiveness when compared with the aforementioned PID benchmark. For the whole section, one of the commonly used control performance measures-i.e., IAE-is adopted to compare each control algorithm.

The fine-tuned PID control behavior can be seen in Figure 8. The control parameters in this PID controller are tuned by the classic Ziegler-Nichols method, which takes a lot of effort; however, its performance can be recognized as a good control benchmark. It should be noted that the emphasis in this research is to propose a practical learning structure that allows the control strategy to learn the interactions with a real transient environment directly (without relying on a simulation model) and finally form a good control behavior. Thus, a conclusion of which algorithm is better than the other cannot be drawn (especially when the driving cycle is known), as it is believed that the control behavior largely depends on the efforts of tuning.

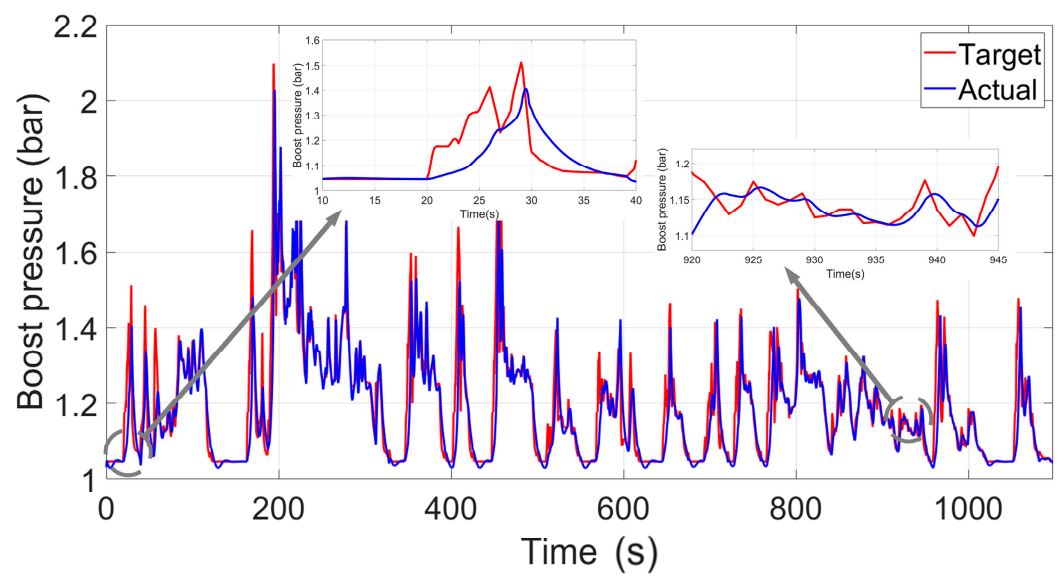

Figure 8. Control behavior of a fine-tuned PID.

The learning curve that shows the cumulated rewards of each episode for the hybrid algorithm, the DDPG algorithm, and the benchmark PID controller can be seen from Figure 9. The trajectory of the DDPG algorithm (which has been published in [15]) illustrates a classical learning process from scratch using model-free DRL algorithms. It is shown that from the beginning of the training that the accumulated rewards for the DDPG agent per episode are extremely low, indicating poor control behavior, and after approximately 40 episodes, the accumulated rewards can be converged slowly to a higher value than that of the benchmark fine-tuned PID controller, indicating a preferable control performance. However, this approach cannot be directly applied on a real plant due to the possible plant damage resulting from its random and not wise control action when the agent has limited experience, especially at the beginning of the training. However, the proposed hybrid algorithm is 
distinct from the DDPG learning curve by offering the control strategy a good action baseline, making it practicable to train the algorithm directly on a real plant.

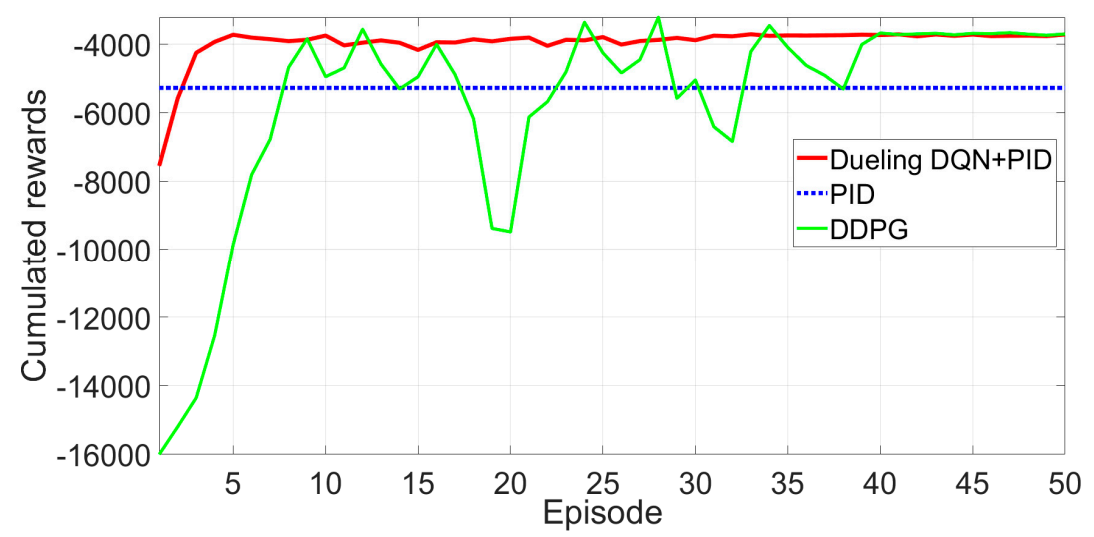

Figure 9. Learning curve for the hybrid algorithm, deep deterministic policy gradient (DDPG) with a fine-tuned Proportion Integration Differentiation (PID) as a benchmark ( ${ }^{*}$ a similar reward function is applied to the system equipped with the PID controller).

To make it clearer, the control behaviors of the DDPG and the hybrid algorithm at the very first training episode are shown in Figure 10. It is shown that the boost pressure of the DDPG algorithm is far away from the target, and due to its random control action (so as to realize extensive exploration), the boost pressure easily exceeds the safety margin. This may be forgiven in the simulation environment, but will most likely damage a real plant in one attempt, not to mention that this kind of model-free DRL algorithm requires a very large number of random actions to achieve good performance. However, for the proposed hybrid algorithm, the boost tracking performance at the very first episode is acceptable, and the boost pressure is well below the safety margin. This is because (1) the action range of the dueling DQN is limited; and (2) the PID acts as a compensator to modify the incorrect action implemented by the dueling DQN at the very first episode. Note that only after approximately five episodes, the hybrid algorithm has already been converged, and the accumulated rewards are basically the same as those achieved by the DDPG algorithm requiring nearly 50 episodes, indicating a superior computational efficiency. Figure 11 shows the control behavior of the proposed hybrid algorithm, and when it is compared with Figure 8, it can be seen that the control behavior based on the proposed hybrid algorithm outperforms that of the PID controller, with the IAE for each being 40.12 and 31.83, respectively.

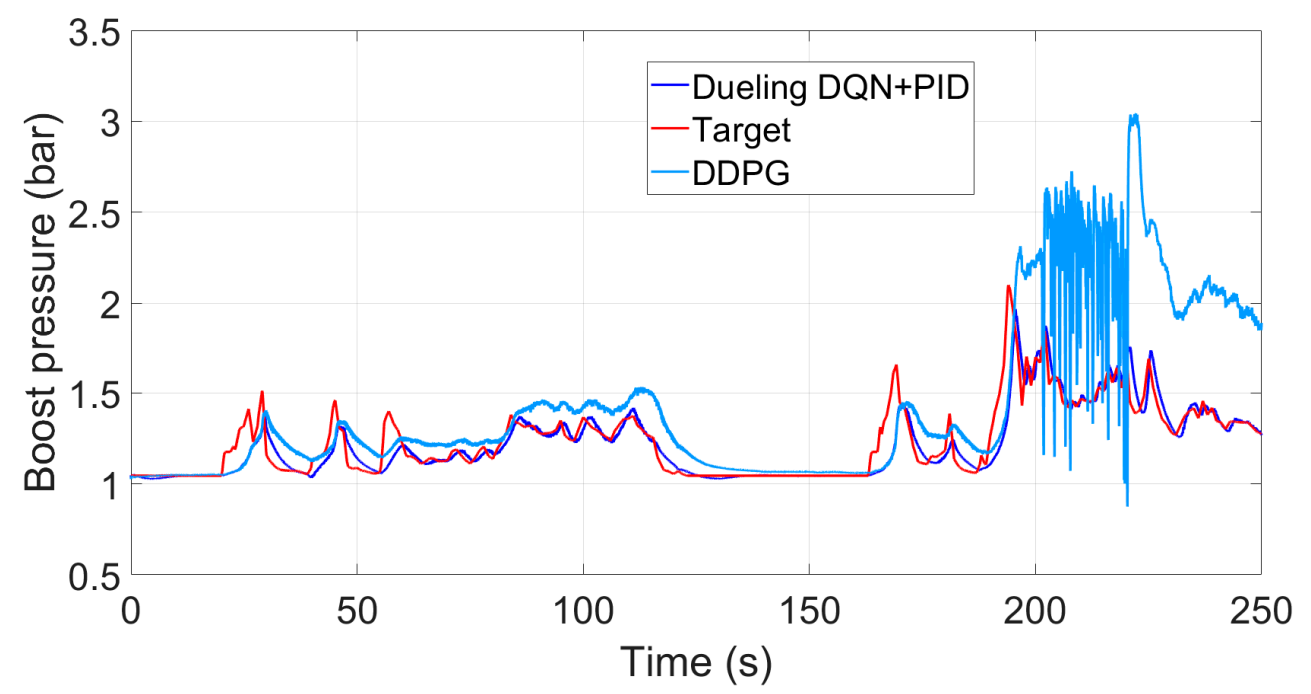

Figure 10. Control behavior comparison between the DDPG and the proposed hybrid algorithm at the very first training episode. 


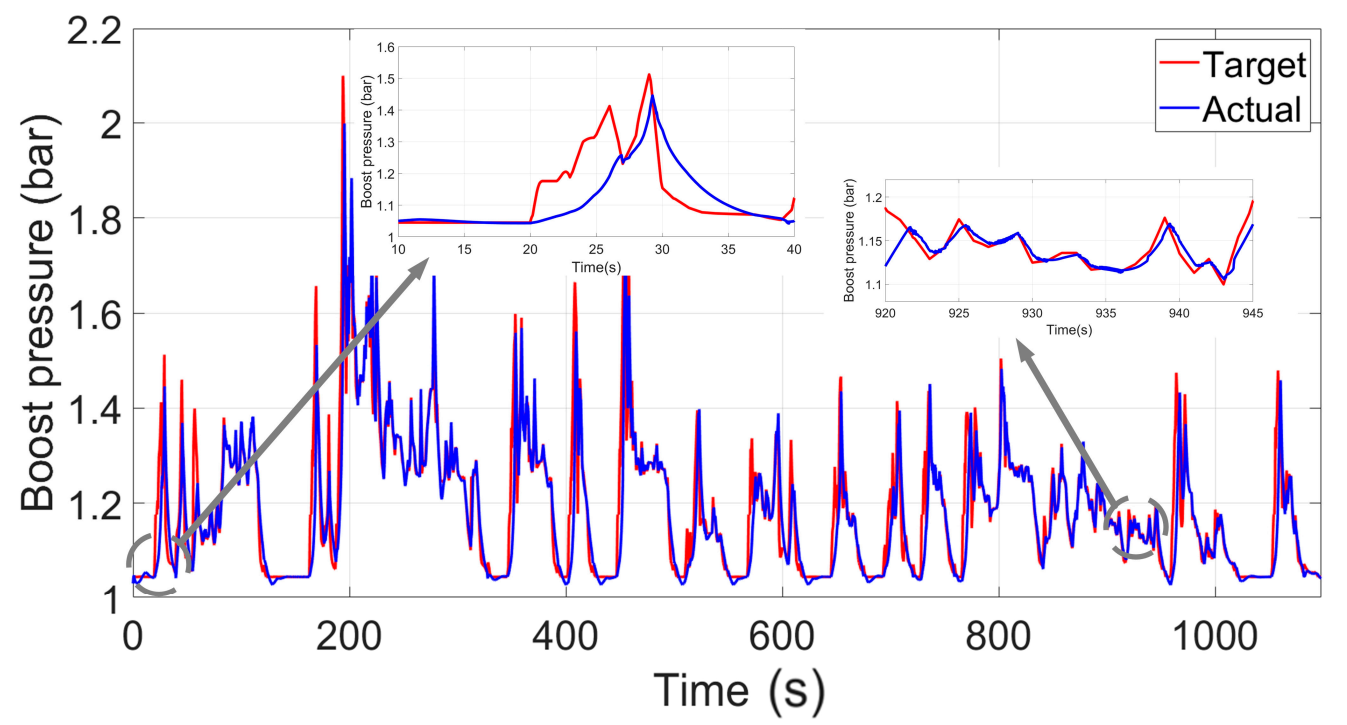

Figure 11. Control behavior of the proposed hybrid algorithm.

In order to show the generalization ability of the proposed hybrid algorithm, as indicated in Figure 12, the control behavior comparison between the fine-tuned PID and the proposed hybrid algorithm using testing dataset is conducted. Compared with the results of the fine-tuned PID controller (the parameters of the PID controller were initially optimized for the whole period of the FTP-72 driving cycle), the average control behavior based on the proposed algorithm performs marginally better, as indicated by the IAE of the PID being 9.84 and that of the proposed algorithm being 8.89. However, the actual boost pressure of the proposed algorithm suffers a high-frequency oscillation compared with the PID benchmark. In order to test its adaptivity, a continuous learning is implemented using a long driving cycle containing this specific driving cycle. After a short time, as shown in Figure 13, the agent is able to follow the target boost better, and the oscillation is almost eliminated, showing the superior adaptiveness of this hybrid algorithm.

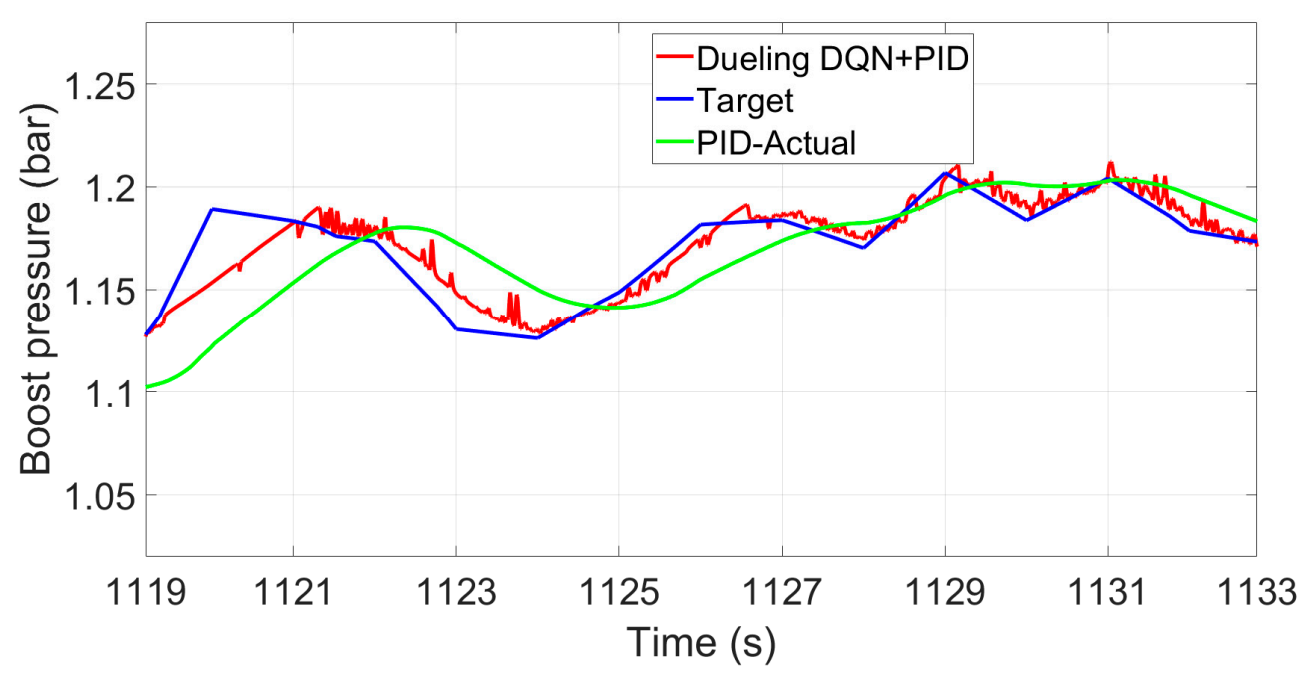

Figure 12. Control behavior comparison between the fine-tuned PID and the proposed hybrid algorithm using the testing dataset. 


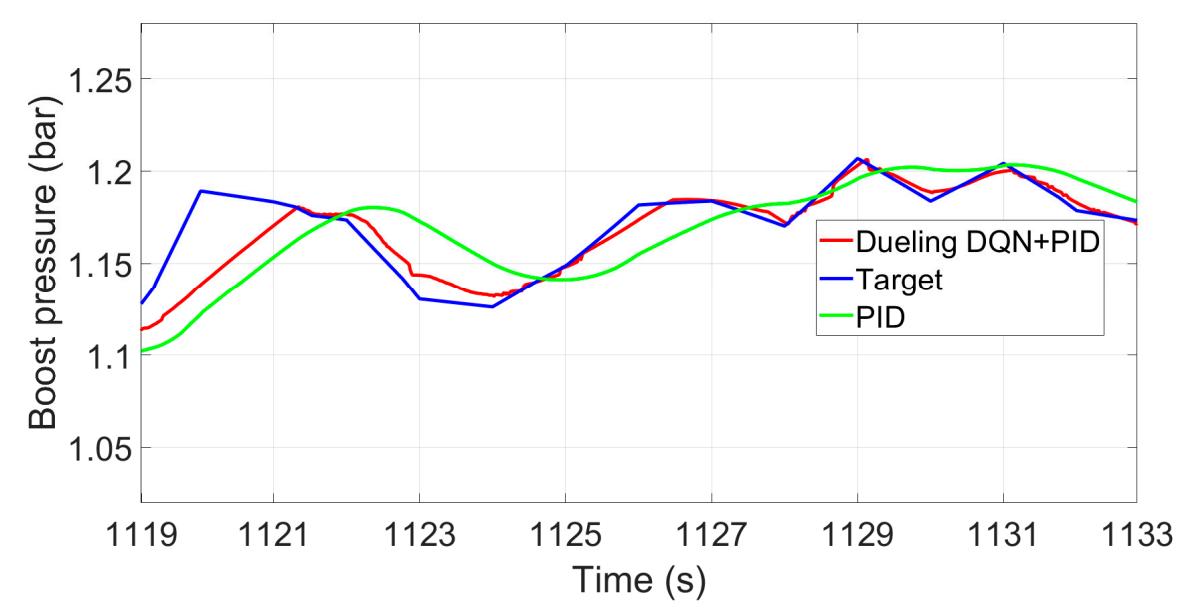

Figure 13. Control behavior after a continuous learning using a long driving cycle containing this specific driving cycle.

Furthermore, by adding another redundant control module, this hybrid method can also improve the system's reliability. This redundancy, unlike most of the other literature, is achieved by enabling DRL algorithms to adapt themselves to the behavior of the system under different PID outputs while trying to maximize the cumulative rewards. This will not only help improve the control behavior when the PID controller behaves decently, but also provide a satisfactory control correction when the PID controller functions abnormally or a total failure occurs. For example, under minor PID issues, the dueling DQN is able to adjust the control parameters autonomously by direct interaction with the environment (thus, a well-behaved controller can be re-developed). Figure 14 simulates this phenomenon by adding a random noise to the PID controller. It is shown that the hybrid algorithm behaves decently well, while the benchmarking PID controller suffers pressure oscillation. For bigger problems, this hybrid architecture can also output a relatively good control behavior, which will help keep the power within the reasonable ranges for the control case of boosted internal combustion engines. This is done by introducing a simple switch in the hybrid controller. To be more specific, when a PID controller failure occurs, a constant value of 0.5 in our study rather than 1 (which is the current industrial practice to protect the engine) is sent to the dueling DQN controller, as can be seen in Figure 7. Without requiring re-learning with the environment (see Figure 15), a good control behavior can still be realized, and it's much better than that of the PID benchmark, whose pressure is well below the target, indicating a lack of power.

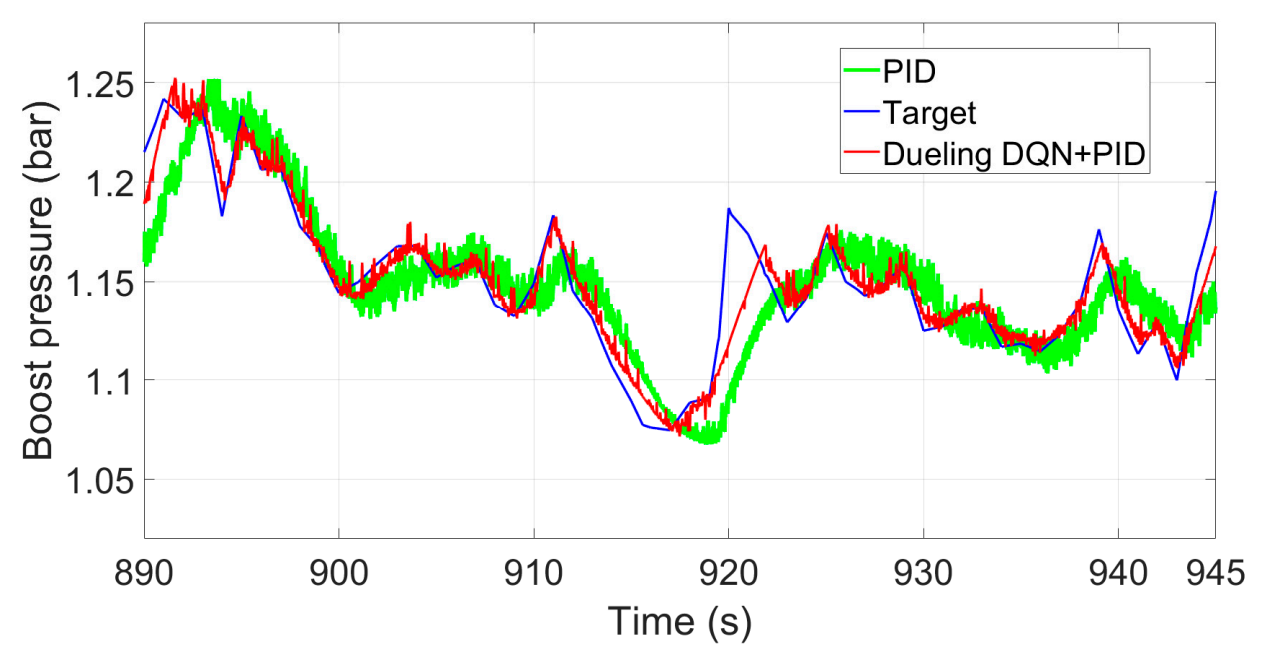

Figure 14. Control behavior between the original PID controller and the hybrid algorithm when the controller is mixed with random noise. 


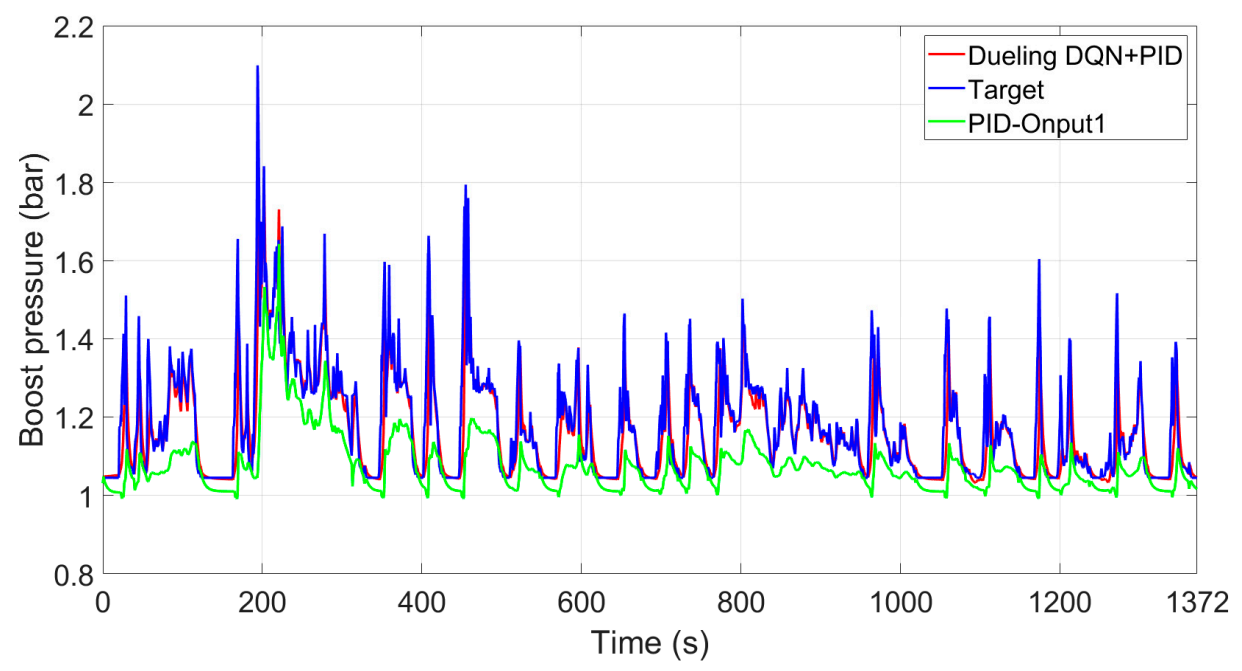

Figure 15. Control behavior between the original PID controller and the hybrid controller when the PID controller fails.

\section{Conclusions}

In this research, a hybrid control algorithm combining a model-free deep reinforcement learning algorithm and a traditional PID controller is proposed. Unlike most of the other DRL studies whose control strategies can only be trained in a simulation environment in order to realize an extensive random exploration while not violating the real plant safety limit, the purpose of this research is to seek a practical method that enables the DRL agent in the real environment to learn the control behavior on its own while adapting to the changing circumstances. By combining both an intelligent dueling DQN and a traditional PID, the proposed hybrid control approach, innovatively allowing the training process of the DRL algorithm that previously can only be carried out in the simulation environment, can now be performed directly on a real plant. This is realized by giving the control strategy some 'guided experience' before learning through making use of an existing fine-tuned PID controller and then allowing DRL control actions to autonomously learn the interaction with the real environment in order to further improve the control performance, based on a relatively good benchmark. Taking the boost control of a VGT-equipped diesel engine as an example, the proposed hybrid algorithm is proven to be able to realize a high level of control performance, generality, and adaptiveness, compared with a fine-tuned PID controller benchmark. Future work may include applying DRL-based parallel architecture or combining model-based RL and the model-free RL techniques to accelerate the training process and improve the final control performance.

Author Contributions: B.H. drafted this paper and provided the overall research ideas. J.L. built the hybrid end-to-end algorithm. S.L. and J.Y. conducted the data analysis. B.H. and J.L. equally contributed to this research work.

Funding: This work is supported by the National Natural Science Foundation of China (Grants No. 51905061), the Natural Science Foundation of Chongqing (Grant No. cstc2019jcyj-msxmX0097), the Science and Technology Research Program of Chongqing Municipal Education Commission (Grant No. KJQN201801124), and the Open Project Program of the State Key Laboratory of Engines (Tianjin University) (Grant No. k2019-02).

Conflicts of Interest: The authors declare no conflict of interest.

\section{References}

1. Li, T.; Wang, B.; Zheng, B. A comparison between Miller and five-stroke cycles for enabling deeply downsized, highly boosted, spark-ignition engines with ultra expansion. Energy Convers. Manag. 2016, 123, 140-152. [CrossRef]

2. Hu, B.; Akehurst, S.; Brace, C. Novel approaches to improve the gas exchange process of downsized turbocharged spark-ignition engines: A review. Int. J. Eng. Sci. 2016, 17, 595-618. [CrossRef] 
3. Hu, B.; Chen, C.; Zhan, Z.; Su, X.; Hu, T.; Zheng, G.; Yang, Z. Progress and recent trends in 48 V hybridisation and e-boosting technology on passenger vehicles-A review. J. Automob. Eng. 2018, 232, 1543-1561. [CrossRef]

4. Hu, B.; Turner, J.W.; Akehurst, S.; Brace, C.; Copeland, C. Observations on and potential trends for mechanically supercharging a downsized passenger car engine:a review. J. Automob. Eng. 2017, 231, 435-456. [CrossRef]

5. Turner, J.W.G.; Popplewell, A.; Patel, R.; Johnson, T.R.; Darnton, N.J.; Richardson, S.; Bredda, S.W.; Tudor, R.J.; Bithell, C.I.; Jackson, R.; et al. Ultra boost for economy: Extending the limits of extreme engine downsizing. SAE Int. J. Engines 2014, 7, 387-417. [CrossRef]

6. Zhao, D.; Winward, E.; Yang, Z.; Stobart, R.; Steffen, T. Characterisation, control, and energy management of electrified turbocharged diesel engines. Energy Convers. Manag. 2017, 135, 416-433. [CrossRef]

7. Feneley, A.J.; Pesiridis, A.; Andwari, A.M. Variable geometry turbocharger technologies for exhaust energy recovery and boosting-A review. Renew. Sustain. Energy Rev. 2017, 71, 959-975. [CrossRef]

8. Zhao, D.; Winward, E.; Yang, Z.; Stobart, R.; Mason, B.; Steffen, T. An integrated framework on characterization, control, and testing of an electrical turbocharger assist. IEEE Trans. Ind. Electron. 2018, 65, 4897-4908. [CrossRef]

9. Oh, B.; Lee, M.; Park, Y.; Won, J.; Sunwoo, M. Mass air flow control of common-rail diesel engines using an artificial neural network. J. Automob. Eng. 2013, 227, 299-310. [CrossRef]

10. Park, I.; Hong, S.; Sunwoo, M. Robust air-to-fuel ratio and boost pressure controller design for the EGR and VGT systems using quantitative feedback theory. IEEE Trans. Control Syst. Technol. 2014, 22, 2218-2231. [CrossRef]

11. Zhang, X.; Sun, L.; Zhao, K.; Sun, L. Nonlinear speed control for PMSM system using sliding-mode control and disturbance compensation techniques. IEEE Trans. Power Electron. 2012, 28, 1358-1365. [CrossRef]

12. Gao, R.; Gao, Z. Pitch control for wind turbine systems using optimization, estimation and compensation. Renew. Energy 2016, 91, 501-515. [CrossRef]

13. Jung, J.W.; Choi, Y.S.; Leu, V.Q.; Choi, H.H. Fuzzy PI-type current controllers for permanent magnet synchronous motors. IET Electr. Power Appl. 2011, 5, 143-152. [CrossRef]

14. Sant, A.V.; Rajagopal, K.R. PM synchronous motor speed control using hybrid fuzzy-PI with novel switching functions. IEEE Trans. Magn. 2009, 45, 4672-4675. [CrossRef]

15. Hu, B.; Yang, J.; Li, J.; Li, S.; Bai, H. Intelligent Control Strategy for Transient Response of a Variable Geometry Turbocharger System Based on Deep Reinforcement Learning. Processes 2019, 7, 601. [CrossRef]

16. Chen, P.; He, Z.; Chen, C.; Xu, J. Control Strategy of Speed Servo Systems Based on Deep Reinforcement Learning. Algorithms 2018, 11, 65. [CrossRef]

17. Sutton, R.S.; Barto, A.G. Reinforcement Learning: An Introduction, 2nd ed.; The MIT Press: Cambridge, MA, USA, 2018; pp. 97-113.

18. Hu, B.; Li, J.; Yang, J.; Bai, H.; Li, S.; Sun, Y.; Yang, X. Reinforcement Learning Approach to Design Practical Adaptive Control for a Small-Scale Intelligent Vehicle. Symmetry 2019, 11, 1139. [CrossRef]

19. Mbuwir, B.V.; Ruelens, F.; Spiessens, F.; Deconinck, G. Battery Energy Management in a Microgrid Using Batch Reinforcement Learning. Energies 2017, 10, 1846. [CrossRef]

20. Liu, T.; Zou, Y.; Liu, D.; Sun, F. Reinforcement Learning-Based Energy Management Strategy for a Hybrid Electric Tracked Vehicle. Energies 2015, 8, 7243-7260. [CrossRef]

21. Shang, X.; Li, Z.; Ji, T.; Wu, P.Z.; Wu, Q. Online Area Load Modeling in Power Systems Using Enhanced Reinforcement Learning. Energies 2017, 10, 1852. [CrossRef]

22. Tan, H.; Zhang, H.; Peng, J.; Jiang, Z.; Wu, Y. Energy management of hybrid electric bus based on deep reinforcement learning in continuous state and action space. Energy Convers. Manag. 2019, 195, 548-560. [CrossRef]

23. Mnih, V.; Kavukcuoglu, K.; Silver, D.; Rusu, A.A.; Veness, J.; Bellemare, M.G.; Graves, A.; Riedmiller, M.; Fidjeland, A.K.; Ostrovski, G.; et al. Human-level control through deep reinforcement learning. Nature 2015, 518, 529-533. [CrossRef] [PubMed]

24. Silver, D.; Aja, H.; Maddison, J.C.; Guez, A.; Sifre, L.; van den Driessche, G.; Schrittwieser, J.; Antonoglou, L.; Panneershelvam, V.; Lanctot, M.; et al. Mastering the game of go with deep neural networks and tree search. Nature 2016, 529, 484-489. [CrossRef] [PubMed] 
25. Silver, D.; Schrittwieser, J.; Simonyan, K.; Antonoglou, I.; Huang, A.; Guez, A.; Hubert, T.; Baker, L.; Lai, M.; Bolton, A.; et al. Mastering the Game of Go without Human Knowledge. Nature 2017, 550, 354-359. [CrossRef] [PubMed]

26. Wang, Z.; Schaul, T.; Hessel, M.; Van, H.H.; Lanctot, M.; Freitas, N.D. Dueling Network Architectures for Deep Reinforcement Learning. arXiv 2016, arXiv:1511.06581.

27. Nikzadfar, K.; Shamekhi, A.H. An extended mean value model (EMVM) for control-oriented modeling of diesel engines transient performance and emissions. Fuel 2015, 154, 275-292. [CrossRef]

28. Kulkarni, T.D.; Narasimhan, K.R.; Saeedi, A.; Tenenbaum, J.B. Hierarchical deep reinforcement learning: Integrating temporal abstraction and intrinsic motivation. In Advances in Neural Information Processing Systems; The MIT Press: Cambridge, MA, USA, 2016; pp. 3675-3683.

29. Yang, G.; Zhang, F.; Gong, C.; Zhang, S. Application of a Deep Deterministic Policy Gradient Algorithm for Energy-Aimed Timetable Rescheduling Problem. Energies 2019, 12, 3461. [CrossRef]

30. Shi, Q.; Lam, H.K.; Xiao, B.; Tsai, S.H. Adaptive PID controller based on Q-learning algorithm. CAAI Trans. Intell. Technol. 2018, 3, 235-244. [CrossRef]

(C) 2019 by the authors. Licensee MDPI, Basel, Switzerland. This article is an open access article distributed under the terms and conditions of the Creative Commons Attribution (CC BY) license (http://creativecommons.org/licenses/by/4.0/). 